\title{
BMJ Open Profile of a population-based diabetic macular oedema study: the Liverpool Eye and Diabetes Study (Sydney)
}

\author{
Gerald Liew, ${ }^{1,2}$ Vincent W Wong, ${ }^{3}$ Mercy Saw, ${ }^{3}$ Tania E Tsang, ${ }^{1}$ Tim Nolan, ${ }^{1}$ \\ Stephen Ong, ${ }^{1}$ I-Van $\mathrm{Ho}^{1,4,5}$
}

To cite: Liew G, Wong VW, Saw M, et al. Profile of a population-based diabetic macular oedema study: the Liverpool Eye and Diabetes Study (Sydney). BMJ Open 2019;9:e021884. doi:10.1136/ bmjopen-2018-021884

- Prepublication history for this paper is available online. To view these files, please visit the journal online (http://dx.doi. org/10.1136/bmjopen-2018021884).

GL and VWW contributed equally.

Received 23 January 2018 Revised 12 October 2018 Accepted 17 October 2018

Check for updates

(c) Author(s) (or their employer(s)) 2019. Re-use permitted under CC BY-NC. No commercial re-use. See rights and permissions. Published by BMJ.

For numbered affiliations see end of article.

Correspondence to

Professor Gerald Liew; gerald_liew@yahoo.com.au

\section{ABSTRACT}

Purpose The population prevalence of diabetic macular oedema (DME) is unclear. Previous estimates have depended on photographic grading of clinically significant macular oedema, which is subjective and has resulted in widely varying estimates. With the advent of optical coherence tomography (OCT), the presence and severity of DME can now be assessed objectively and accurately. Methods The Liverpool Eye and Diabetes Study (LEADS) is a cross-sectional population-based study of patients with type 1 and type 2 diabetes in a multi-ethnic region of Sydney, Australia, to determine the population prevalence of OCT-defined DME, how this varies by ethnicity and association with systemic factors. This report describes the rationale, methodology and study aims.

Results To date 646 patients out of an expected sample size of 2000 have been recruited. Baseline data are presented for patients with type $1(n=75,11.8 \%)$ and type 2 ( $n=562,88.2 \%)$ diabetes recruited to date. Patients with type 1 diabetes were younger (39.5vs60.7 years), with longer duration of diabetes (18.1vs11.7 years), slightly worse glycaemic control (HbA1c 9.0vs8.3), and less likely to have hypertension (30.7vs71.4\%), hypercholesterolaemia (33.3vs74.6\%) and obesity (31.1vs51.5\%, respectively, all $p<0.05$ ).

Conclusions The LEADS will provide objective estimates of the population prevalence of DME, how this varies with ethnicity and associations with systemic disease.

\section{INTRODUCTION}

Diabetic eye disease is one of the leading causes of blindness among working age adults in Australia and other developed countries. ${ }^{1}$ Much of the blindness from diabetic eye disease is preventable with early detection and treatment. Diabetic macular oedema (DME) or thickening of the central retina, is the main cause of visual loss in diabetic eye disease. $^{2}$ DME has traditionally been treated with focal laser therapy but more recently intravitreal anti-vascular endothelial growth factor (anti-VEGF) agents have been introduced which have dramatically improved treatment outcomes.

Despite being a major cause of visual loss, the prevalence of DME in patients with

\section{Strengths and limitations of this study}

This study used optical coherence tomographydefined diabetic macular oedema as an objective measure to determine the prevalence of the condition in a multi-ethnic population.

- Data on ethnicity, some socioeconomic indicators, medical comorbidities and other factors were collected.

Patients with gestational diabetes were excluded.

diabetes is not known with certainty. This is because most published estimates of the prevalence of macular oedema were determined from photographs of the retina, a form of macular oedema known as 'clinically significant macular oedema' (CSME). Epidemiological studies based on this method have reported a range of DME prevalence in the general diabetic population of between $3 \%$ and $14 \% .{ }^{2-4}$ Interestingly there appears to be an ethnic variation, with higher prevalence in American Blacks and Hispanics, followed by American Chinese and lowest rates in American Whites reported in studies such as the Multi-Ethnic Study of Atherosclerosis (MESA).$^{4-6}$ This may be related to differences in response to conventional risk factors such as hyperglycaemia and hypertension, different body habitus and lifestyle factors such as diet, smoking, exercise, and genetic and epigenetic factors. ${ }^{47}$ There is no comparable multi-ethnic data in Australia showing the prevalence rates among different ethnic groups. Indigenous Australians are known to have far higher rates of diabetic retinopathy and this has led to focused efforts to reduce the burden of blindness from the disease in this vulnerable group. Similar data are required for other ethnic groups to identify which groups are most at risk and to plan interventions accordingly.

Previous estimates of CSME prevalence were useful when focal laser therapy was the 
only available treatment for macular oedema but are now outdated and of limited clinical utility in the era of antiVEGF agents. Further, highly sensitive new imaging technology such as optical coherence tomography (OCT) is now available which can image the centre of the retina at an almost histological level and both detect and quantify the amount of DME. Few studies have reported the prevalence of OCT detected DME and those that have were all clinic based surveys of limited use in estimating the true population prevalence. ${ }^{8-11}$ These surveys suggest a markedly higher prevalence than photographic detection, with rates of between $30 \%$ and $40 \%$ in patients with type 2 diabetes. ${ }^{8912}$ The more sensitive OCT detection of DME may also help resolve the highly clinically relevant question of which systemic conditions increase the risk of developing DME, as photographic studies using the less accurate CSME definition have reported inconsistent associations with conditions other than the well-known risk factors of hyperglycaemia and hypertension. ${ }^{13-16}$

There is thus a need for accurate data on the population prevalence of OCT-defined DME and associations with systemic diseases. We are conducting a population-based cohort study to obtain this data and report on the methodology here.

\section{MATERIALS AND METHODS \\ Study design}

The Liverpool Eye and Diabetes Study (LEADS) is a non-interventional, population-based observational cross-sectional study based in the Liverpool local government area of Sydney, New South Wales, Australia. The study is being conducted according to the tenets of the Declaration of Helsinki.

\section{Study population}

The catchment area is the South Western Sydney Local Area Health District with an estimated 8000 subjects with diabetes. Residents who live in this area are from diverse ethnic backgrounds and between 2000 and 2011, the prevalence of diabetes increased substantially by $158 \%{ }^{17}$ The prevalence of DME in this population is unknown. The population living in the region of interest is comparable to, and representative of those living in the broader Liverpool (Sydney) area in terms of gender ratio, mean age and median income ${ }^{17}$ Patients are recruited from the Liverpool Hospital Diabetes and Endocrinology Service, general practitioners (GPs), private endocrinologists and private optometrists. A standardised referral form is used to facilitate uniform collection of data from referrers such as demographics, medications, medical history. Recruitment commenced in April 2015 and is ongoing.

\section{Patient and public involvement}

Patients' experiences and observations as relayed to the study team members formed the basis of the study rationale. Patients will be informed of the study findings through local newsletters. The public was not involved in the planning of this study.

\section{Eligibility criteria}

1. Patients with diabetes mellitus: type 1, type 2 diabetes or diabetes due to other causes (monogenic diabetes syndrome, pancreatic disease or drug induced diabetes). Type 1 and type 2 were defined according to the referring endocrinologist or GP; if these diagnoses were not available, type 1 was defined as onset before age 40 and current use of insulin.

2. Aged over 18 years.

3. Able to provide informed consent.

4. Residing in the South Western Sydney Local Health District (SWSLHD).

\section{Exclusion criteria}

1. Age $<18$ years.

2. Pregnant women or women with gestational diabetes mellitus.

3. Unable to provide informed consent.

4. Residing outside of SWSLHD.

\section{Main aims and hypotheses}

Aim 1. The primary aim of this study is to determine the prevalence of OCT-defined DME in a multi-ethnic population with diabetes in South Western Sydney, NSW, Australia. Results will be presented for different ethnic groups including persons of European, East Asian (Vietnamese, Chinese), South Asian (including Indian, Pakistani, Sri Lankan) and Arabic (including Lebanese, Iranian) backgrounds.

Hypothesis. The prevalence of DME is higher in an ethnically diverse population compared with Anglo-European populations.

Aim 2. A secondary aim of the study is to examine systemic associations of DME.

Hypothesis. With more sensitive OCT detection of DME, this study will be able to detect associations with systemic risk factors such as glycaemic control, blood pressure, dyslipidaemia, nephropathy and neuropathy.

Aim 3. Another secondary aim is to examine differences in ethnic presentations of diabetic retinopathy lesions (microaneurysms, haemorrhages, neovascularisation).

Hypothesis. The prevalence of diabetic retinopathy lesions is higher in persons of South Asian, East Asian and Middle-Eastern origin compared with persons of Anglo-European origin.

\section{Examination procedures}

1. Visual acuity. Patients attended for a 90 min consultation and had their best corrected visual acuity measured, including pinhole vision using Early Treatment Diabetic Retinopathy Study (ETDRS) charts. Both pupils were dilated with $1 \%$ tropicamide and $10 \%$ phenylephrine as recommended by National Health and Medical Research Council (NHMRC) guidelines. ${ }^{18}$

2. Medical history. The research officer conducted a medical interview and filled in a standard questionnaire 
obtaining information on demographics such as age, gender, self-reported ethnicity, ocular and medical history, other complications of diabetes (both micro and macrovascular disease), and cardiovascular risk factors. All medication conditions were self-reported and hypertension and hyperlipidaemia were inferred from the medications list provided by the participants. Medications were confirmed with the referral medication list. Obesity was defined as body mass index (BMI) $\geq 30 \mathrm{~kg} / \mathrm{m}^{2}$. Neuropathy was defined from history provided by the treating endocrinologist or GP. Blood pressure, height and weight were measured during the consultation.

3. Retinal imaging. All patients recruited had digital retinal photography using a standardised protocol. After pupil dilation, fundus photography was performed with a digital non-mydriatic retinal camera (Canon CR-DGi with a 20Diopter SLR backing, Canon, Japan). Photographs included ETDRS standard fields 1 (centred on the optic disc) and 2 (centred on the fovea). This photographic method has been shown to have high sensitivity and specificity for detecting diabetic retinopathy ${ }^{19}$ and is used in the UK NHS National Diabetic Eye Screening programme. ${ }^{20}$ Fundus autofluorescence and red free images were also taken. A Heidelberg spectral domain OCT took macula centred high density raster scans through the fovea. OCT images were obtained on the same day, after pupil dilation. The Spectralis HRA+OCT with viewing module V.5.1.2.0 (Heidelberg Engineering, Heidelberg, Germany) was used to acquire Spectral Domain Optical Coherence Tomography (SD-OCT) images. The SDOCT protocol included a dense horizontal linear scan centred on the fovea and the HEYEX software interface (V.1.6.2.0; Heidelberg Engineering) was used for registration and evaluation. DME was defined as cystic spaces in at least two consecutive raster scans; CSME was defined from fundus photographs according to ETDRS criteria. ${ }^{21}$ The reproducibility of the Heidelberg SD-OCT measurements is reported to be higher than most other OCT machines. ${ }^{22}$ OCT procedure followed the APOSTEL guidelines and further details are available on request. ${ }^{23}$ Intraocular pressure was measured with a Tono-pen.

4. Medical review. Images were reviewed and slit lamp examination performed by a consultant ophthalmologist. Any patients with abnormalities requiring urgent treatment (eg, DME and reduced vision, CSME, proliferative diabetic retinopathy etc) were referred for treatment according to NHMRC guidelines. Presence and severity of cataract, corneal abnormalities, vitreous posterior vitreous detachment and peripheral retinal lesions were documented. B-scan ultrasound was performed if dense cataract precludes adequate retinal examination.

5. Diabetic retinopathy grading. At completion of the study, the modified Wisconsin protocol will be used for diabetic retinopathy grading. ${ }^{24}$ Diabetic retinopathy will be considered present if any characteristic lesion as defined by the ETDRS severity scale is present: microaneurysms, haemorrhages, cotton wool spots, intraretinal microvascular abnormalities, hard exudates, venous beading and new vessels. For each eye, a retinopathy severity score will be assigned according to a scale modified from the Airlie House classification system.

6. Blood specimen collection and laboratory investigations: $5-10 \mathrm{~mL}$ of venous blood were collected from each subject for the purpose of (1) DNA extraction and genotyping, (2) collection of plasma and serum for assessment of novel blood biomarkers and metabolites and (3) for assessment of glycated haemoglobin (HbA1c), serum creatinine (including estimated glomerular filtration rate), lipid studies (during fasting state) and spot urine albumin-creatinine ratio.

\section{Expected patient numbers and power calculations}

The study is powered to detect a $4 \%$ difference in DME prevalence between different ethnic groups. This represents a clinically significant difference as previous smaller studies suggest a range of between 3\% and 14\% in white populations..$^{2-4}$ Assuming a baseline DME prevalence of $10 \%, 25 \%$ of the sample being of European, South Asian, East Asian and Middle Eastern ethnicity respectively, power of $80 \%$ and false positive rate of $5 \%$, $\mathrm{n}=3000$ participants in total will need to be recruited to detect this difference. This sample size is larger than that of the MESA that reported contemporary ethnic differences prevalence of diabetic retinopathy and DME $(n=778) .{ }^{24}$ The sample represents $30 \%$ of the total population of patients with diabetes in the defined population postcode.

\section{Statistical analysis}

DME will be described using qualitative (present, absent) and quantitative (central subfield thickness (CST) in $\mu \mathrm{m}$ ) variables, and will be reported as $\%$ and mean $( \pm \mathrm{SD})$ respectively, in the whole population studied, and by ethnic group. Differences between ethnic groups will be assessed using statistical tests such as $\chi^{2}$ test for categorical variables and t-test for continuous variables. Multivariable logistic regression models will be constructed with age, gender, $\mathrm{HbA1c}$, duration of diabetes and other relevant risk factors (including ethnicity, hypertension, hyperlipidaemia, obesity, smoking history and various anti-hyperglycaemic medications) as predictors of the outcome variable (DME). STATA software, V.15 will be used in the analyses.

\section{RESULTS}

As of December 2016, 646 patients with diabetes had been seen as part of LEADS. Of these, 562 had type 2 diabetes $(88.2 \%)$. The baseline characteristics of the study cohort are shown in table 1 . Within the study cohort, the mean age was 58.2 years with participants having a mean duration of diabetes of 12.5 years and a prevalence of 
Table 1 Baseline characteristics of initial 646 patients recruited to Liverpool Eye and Diabetes Study (both type 1 and type 2 diabetes)

\begin{tabular}{|c|c|c|c|}
\hline & Total $n=646$ & Male $n=367$ & Female $n=279$ \\
\hline Age (mean $\pm S D$; in years) & $58.2( \pm 14.4)$ & $58.9( \pm 13.6)$ & $57.3( \pm 15.3)$ \\
\hline $\mathrm{BMI}\left(\mathrm{mean} \pm \mathrm{SD} ; \mathrm{kg} / \mathrm{m}^{2}\right)$ & $30.9( \pm 7.4)$ & $30.1( \pm 6.7)$ & $31.9( \pm 8.1) p<0.01$ \\
\hline$<25$ & $22.4( \pm 2.0)$ & $22.6( \pm 1.9)$ & $22.2( \pm 2.2)$ \\
\hline $25-30$ & $27.4( \pm 1.5)$ & $27.4( \pm 1.6)$ & $27.3( \pm 1.4)$ \\
\hline$>30$ & $36.8( \pm 6.1)$ & $36.4( \pm 5.2)$ & $37.2( \pm 6.9)$ \\
\hline Duration of diabetes (mean $\pm S D$; in years) & $12.5( \pm 9.7)$ & $12.3( \pm 9.4)$ & $12.8( \pm 10.0)$ \\
\hline Mean HbA1c n (\%) (with \pm SD) & $8.4( \pm 2.2)$ & $8.6( \pm 2.4)$ & $8.1( \pm 2.0) p<0.01$ \\
\hline International Federation of Clinical Chemistry (mmol/mol) & 68.3 & 70.5 & 65.0 \\
\hline $\mathrm{mmol} / \mathrm{L}$ & 10.8 & 11.1 & 10.3 \\
\hline$<7 \%$ & $6.4( \pm 0.5)$ & $6.4( \pm 0.5)$ & $6.4( \pm 0.5)$ \\
\hline $7 \%-9 \%$ & $7.8( \pm 0.6)$ & $7.8( \pm 0.6)$ & $7.8( \pm 0.6)$ \\
\hline$>9 \%$ & $11.1( \pm 1.9)$ & $11.4( \pm 2.0)$ & $10.8( \pm 1.7)$ \\
\hline $\begin{array}{l}\text { SBP }(m m ~ H g) \\
\text { DBP }(m m ~ H g)\end{array}$ & $\begin{array}{l}134.5( \pm 20.5) \\
76.3( \pm 11.8)\end{array}$ & $\begin{array}{l}135.2( \pm 19.3) \\
77.3( \pm 11.9)\end{array}$ & $\begin{array}{l}133.5( \pm 21.9) \\
74.9( \pm 11.6)\end{array}$ \\
\hline \multicolumn{4}{|l|}{ Smoking status n (\%) } \\
\hline Non-smoker & $332(51.4)$ & $154(42.1)$ & $178(63.7) p<0.001$ \\
\hline Ex-smoker & $211(32.6)$ & $145(39.3)$ & $66(23.7)$ \\
\hline Current smoker & $103(16.0)$ & $68(18.6)$ & $35(12.6)$ \\
\hline Hypertension n (\%) & $430(66.5)$ & $255(69.5)$ & $174(62.2) p<0.05$ \\
\hline Hypercholesterolaemia n (\%) & $450(69.7)$ & $255(69.5)$ & $195(69.9)$ \\
\hline Obesity n (\%) & $312(48.3)$ & $162(44.2)$ & 154 (55.1) $p<0.05$ \\
\hline
\end{tabular}

Obesity is defined as $\mathrm{BMI} \geq 30$. Statistically significant differences are marked. Other comparisons were not statistically significant at $p<0.05$. $\mathrm{BMI}$, body mass index; DBP, diastolic blood pressure; SBP, systolic blood pressure.

hypertension and hypercholesterolaemia, $66.5 \%$ and $69.7 \%$, respectively. On average, the subjects were obese and had a BMI above $30 \mathrm{~kg} / \mathrm{m}^{2}$ and suboptimal glycaemic control, with mean HbAlc of $8.4 \%(68 \mathrm{mmol} / \mathrm{mol})$.

Differences in baseline characteristics between those with type 1 and type 2 diabetes are summarised in table 2 . Those with type 2 diabetes were significantly older, had shorter duration of diabetes, higher BMI and higher systolic blood pressure compared with their type 1 diabetes counterparts. They similarly had significantly higher rates of hypertension, hypercholesterolaemia and obesity than those with type 1 diabetes. They did however have better glycaemic control with lower HbAlc, and fewer were current smokers compared with those with type 1 diabetes.

\section{DISCUSSION}

DME is one of the leading causes of treatable blindness in working age adults with diabetes, but the prevalence remains uncertain. This may be due to variations in how DME is defined and measured. The mean age of our cohort was 58.2 years and $56.8 \%$ of our cohort was male, mean HbA1c was $8.4 \%$ and $50 \%$ of the cohort were ex-smokers or current smokers. These results are similar to those from other Australian cohorts of patients with diabetes, ${ }^{25}$ suggesting our population is representative and recruitment is not biased.

Until recently, studies on the prevalence of diabetic retinopathy have used photographic assessment ('CSME') which may underestimate the prevalence by threefold or more. $^{8912}$ A 2014 cross-sectional analysis of 1038 participants aged 40 or older with diabetes in the US National Health and Nutrition Examination Survey (NHANES) found weighted prevalence of CSME was 3.8\% based on NHANES digital grading protocol of fundus photography. ${ }^{6}$ This contrasts with a UK study of electronic medical records from 76127 diabetic patients managed by the UK hospital eye service which found CSME present in $15.8 \%-18.1 \%$ of eyes, involving the central macula in $8.7 \%-10.0 \%$ of eyes. ${ }^{26}$ This finding is more in line with those found in the Veteran Affairs Diabetes Trial that observed CSME prevalence of $10.0 \%$ based on 7-field stereo fundus photographs in 1268 type 2 diabetic patients. ${ }^{7}$ Furthermore, a 2012 meta-analysis of 35 population-based studies in the USA, Australia, Europe and Asia from 1980 to 2008 found prevalence of CSME of $7.48 \%$ among individuals with both type 1 and type 2 diabetes. ${ }^{27}$

In contrast, a 2013 Australian study of patients from hospital clinics found CSME prevalence of $24.7 \%$ (413). ${ }^{8}$ Of those with type 1 diabetes, prevalence of CSME was 
Table 2 Comparison of baseline factors between patients with type 1 and type 2 diabetes

\begin{tabular}{|c|c|c|c|}
\hline & $\begin{array}{l}\text { Type } 1 \text { diabetes } \\
(\mathrm{n}=\mathbf{7 5}, \mathbf{1 1 . 8 \% )}\end{array}$ & $\begin{array}{l}\text { Type } 2 \text { diabetes } \\
(\mathrm{n}=562,88.2 \%)\end{array}$ & $P$ value \\
\hline Age (mean $\pm S D$; in years) & $39.5( \pm 16.4)$ & $60.7( \pm 12.1)$ & $\mathrm{p}<0.001$ \\
\hline $\mathrm{BMI}\left(\mathrm{mean} \pm \mathrm{SD} ; \mathrm{kg} / \mathrm{m}^{2}\right)$ & $28.1( \pm 8.3)$ & $31.3( \pm 7.1)$ & $p=0.001$ \\
\hline$<25$ & $21.3( \pm 2.2)$ & $22.6( \pm 1.9)$ & $p=0.018$ \\
\hline $25-30$ & $26.9( \pm 1.3)$ & $27.5( \pm 1.6)$ & $\mathrm{p}=0.033$ \\
\hline$>30$ & $36.2( \pm 10.5)$ & $36.8( \pm 5.6)$ & $p>0.05$ \\
\hline Duration of diabetes (mean $\pm S D$; in years) & $18.1( \pm 13.7)$ & $11.7( \pm 8.7)$ & $\mathrm{p}<0.001$ \\
\hline $\mathrm{HbA1c}(\% \pm \mathrm{SD})$ & 74.9 & $8.3( \pm 2.2)$ & $p<0.023$ \\
\hline International Federation of Clinical Chemistry $(\mathrm{mmol} / \mathrm{mol})$ & 74.9 & 67.2 & \\
\hline In mmol/L & 11.7 & 10.6 & \\
\hline$<7 \%$ & $6.5( \pm 0.6)$ & $6.4( \pm 0.5)$ & $\mathrm{p}>0.05$ \\
\hline $7 \%-9 \%$ & $7.9( \pm 0.5)$ & $7.8( \pm 0.6)$ & $p>0.05$ \\
\hline$>9 \%$ & $11.4( \pm 2.0)$ & $11.1( \pm 1.9)$ & $p>0.05$ \\
\hline $\mathrm{SBP}(\mathrm{mm} \mathrm{Hg})$ & $124.5( \pm 17.5)$ & $135.9( \pm 20.4)$ & $p<0.001$ \\
\hline $\mathrm{DBP}(\mathrm{mm} \mathrm{Hg})$ & $76.3( \pm 11.2)$ & $76.3( \pm 11.9)$ & $p>0.05$ \\
\hline \multicolumn{4}{|l|}{ Smoking status \% (n) } \\
\hline Non-smoker & $58.7(44)$ & $50.9(286)$ & $\mathrm{p}=0.007$ \\
\hline Ex-smoker & $17.3(13)$ & $34.2(192)$ & \\
\hline Current smoker & $24.0(18)$ & $14.9(84)$ & \\
\hline Hypertension \% within diabetes type (n) & $30.7(23)$ & $71.4(400)$ & $p<0.001$ \\
\hline Hypercholesterolaemia \% (n) & $33.3(25)$ & $74.6(415)$ & $\mathrm{p}<0.001$ \\
\hline Obesity \% (n) & $31.1(19)$ & 51.5 (236) & $\mathrm{p}=0.011$ \\
\hline
\end{tabular}

Obesity is defined as $\mathrm{BMI} \geq 30$.

$\mathrm{BMI}$, body mass index.

$14.5 \%$ (59 participants) and $28.1 \%$ prevalence (354 participants) in those with type 2 diabetes, determined by slit lamp biomicroscopy. The population sample was predominantly comprised of Caucasian participants.

In recent years, clinical assessment of DME has shifted from fundus biomicroscopy to OCT imaging. A 2011 Cochrane meta-analysis investigating the accuracy of OCT assessment for detecting DME or CSME examined nine studies with 768 participants and 1325 eyes. Data pooled from eight of these studies determined that prevalence of DME determined from OCT ranged from $19 \%$ to $65 \%$ (median $50 \%$ ) based on median central retinal thickness cut-off of $250 \mu \mathrm{m}$ (range $230 \mu \mathrm{m}-300 \mu \mathrm{m}$ ). ${ }^{9}$ A 2016 study compared the prevalence of DME based on monocular fundus photography with OCT. ${ }^{28}$ They examined 246 eyes of 158 participants comparing fundus photograph prevalence of CSME based on MESA and NHANES definitions with OCT prevalence based on central subfield thickness (CST) measurements. Using OCT, DME was observed in $21.1 \%$ of participants and CSME in $21.3 \%$ of participants. These values were significantly lower than rates estimated by both MESA and NHANES definitions, though NHANES prevalence rates were more closely approximated. This suggests photography can overestimate prevalence of DME compared with definitions based on CST on OCT.

A 2004 study examined the efficacy of contact lens biomicroscopy compared with OCT in detecting diabetic foveal oedema. In their sample of 172 eyes from 95 patients with varying levels of retinopathy (including no retinopathy), central macular oedema as defined by foveal thickening $(>200 \mu \mathrm{m})$ was detected by OCT in 72 eyes $(42 \%)$. However, this high prevalence may reflect a sample bias, as study participants consisted of a 'convenience' cohort of any patients seen at the Retinal Vascular Center with diabetic retinopathy who met inclusion criteria and were seen by the study investigators.

The question of whether the occurrence of DME differs among ethnic groups has not been clearly determined. There have been reported variations between ethnic groups in prevalence studies. A US cross-sectional study of patients participating in MESA has shown significantly higher prevalence of CSME in blacks $(11.1 \%)$ and Hispanics $(10.7 \%)$ than in Chinese $(8.9 \%)$ and whites $(2.7 \%) .{ }^{24}$ Data from the NHANES study had lower prevalences of $8.4 \%$ among non-Hispanic blacks, $5.1 \%$ among Hispanics and $2.6 \%$ among whites, though the trend was similar with prevalence of CSME being approximately 
three times higher among non-Hispanic blacks than the non-Hispanic white population. ${ }^{6}$ However, Chinese studies have shown much lower rates of CSME at 3.5\% and $2.6 \%$ in rural and urban areas respectively. ${ }^{3}$ Additionally, a UK study comparing retinopathy in 421 South Asians with 614 white Europeans found significantly different age-standardised and sex-standardised prevalences of maculopathy between the two ethnic groups ( $14.4 \%$ and $8.8 \%$ respectively, $10 \%$ overall prevalence) (based on photographs). ${ }^{29}$

The association between lipid levels and DME has been equivocal. A 2011 Australian study of 500 participants showed associations between serum lipids and CSME. Those with higher total, Low Density Lipoprotein (LDL)and non-High Density Lipoprotein (HDL)-cholesterol were more likely to have CSME. ${ }^{30}$ However, serum lipids were not associated with diabetic retinopathy, mild or moderate DME or macular thickness. This finding was also seen in a 2010 cross-sectional study of 1414 diabetic subjects in India of which a third had CSME, which was associated with high total cholesterol, HDL and LDL. ${ }^{15}$ Among a type 1 diabetes prospective study cohort total cholesterol to HDL ratio was significantly associated with CSME. ${ }^{13}$

In contrast, analysis of data taken from 730 participants in the Wisconsin Epidemiologic Study of Diabetic Retinopathy found that neither the level of oxidised LDL at the start of the 24-year follow-up period nor changes over time were associated with incidence of proliferative diabetic retinopathy, macular oedema (CSME) or worsening of retinopathy. ${ }^{31}$ A study in type 2 diabetic patients with a smaller sample size similarly found no correlation between serum lipids and CSME. ${ }^{14}$

Markers of nephropathy have similarly had inconsistent associations with CSME. A 2015 analysis of data from the German/Austrian database of 64784 patients with type 2 diabetes found the presence of macroalbuminuria increased the risk of CSME by $177 \%$ and both micro and macroalbuminuria were strong risk predictors of severe retinopathy. ${ }^{32}$ Similar associations were seen between nephropathy and CSME in studies with the markers of nephropathy being microalbuminuria (OR of 14.23$)^{15}$ and increased urinary-albumin excretion. ${ }^{16}$ However, an Australian study of 263 Caucasian patients with type 2 diabetes in a clinic-based cross-sectional study found no association between DME (based on retinal photographs graded with the modified Airlie House classification and further confirmed with OCT) and renal dysfunction as measured by Estimated Glomerular Filtration Rate (eGFR), though lower eGFR was associated with presence and severity of diabetic retinopathy. ${ }^{33}$ This lack of association was also seen in a study where the measure of renal dysfunction was urinary-albumin excretion rates. ${ }^{14}$ The association between CSME and nephropathy is equally uncertain in type 1 diabetic patients, with one study of 656 type 1 diabetics finding no association between CSME and urinary-albumin excretion and another finding no correlation between overt nephropathy and CSME. ${ }^{16}$
In the past 20 years, a number of new classes of diabetes medications, including glucagon like peptide-1 agonists, dipeptidyl peptidase four inhibitors as well as sodium glucose co-transporter two inhibitors had become available. While the impact of these new agents on cardiovascular events has been scrutinised in large clinical trials, their effects on the development and progression of diabetic retinopathy/DME are often not evaluated. Furthermore, in the management of type 1 diabetes, the use of subcutaneous insulin pump and other new tools such as continuous glucose monitoring system has had a great impact on glycaemic control. ${ }^{34-36}$ LEADS may be able to provide some insight as to the relationship between these new diabetes management strategies and diabetic retinopathy/DME. Finally, the relationship between obesity and diabetic retinopathy/DME is not clear, and studies had shown conflicting results. ${ }^{37-39}$ With the prevalence of obesity reaching an epidemic level in south-western Sydney, the impact of obesity on DME can also be assessed in LEADS.

Strengths of this LEADS study include the population-based sampling area with high diabetes prevalence and a multi-ethnic mix. This provides an opportunity to determine ethnic differences in diabetic retinopathy and DME and relate these to lifestyle factors. Objective assessment of DME will also reduce measurement error and improve precision of estimates. This will increase power to detect associations with risk factors and clarify which systemic conditions, in addition to glycaemic control and blood pressure, are associated with DME. This may help clarify the gaps in knowledge and uncertainties listed above.

In summary, the LEADS study will provide accurate and contemporary population-based data on the prevalence of DME in a multi-ethnic region. It will also test hypotheses regarding differences in susceptibility to DME in different ethnic groups, and associated risk factors. The data are important for planning of service provision and in public health efforts to reduce blindness from diabetic eye disease.

\section{Author affiliations}

${ }^{1}$ South West Retina, Retina Associates, Liverpool, New South Wales, Australia ${ }^{2}$ Centre for Vision Research, Westmead Millennium Institute of Medical Research, University of Sydney, Sydney, New South Wales, Australia

${ }^{3}$ Liverpool Diabetes Collaborative Research Unit, Ingham Institute of Applied Science, Sydney, New South Wales, Australia

${ }^{4}$ Save Sight Institute, University of Sydney, Sydney, New South Wales, Australia ${ }^{5}$ Australian School of Advanced Medicine, Macquarie University, Sydney, New South Wales, Australia

Contributors GL, VW, SO, TN and IH conceived the hypotheses and study design. GL, VW, IH, MS and TT collected the data. MS performed the analyses. GL and VW wrote the first draft. GL, VW, SO, TN, IH, MS and TT reviewed and edited the final version.

Funding NHMRC Early Career Fellowship Grant APP1073530 to Gerald Liew.

Competing interests None declared.

Patient consent for publication Not required.

Ethics approval Sydney South West Area Health Human Research Ethics Committee and the University of Sydney Human Research Ethics Committee.

Provenance and peer review Not commissioned; externally peer reviewed. 
Open access This is an open access article distributed in accordance with the Creative Commons Attribution Non Commercial (CC BY-NC 4.0) license, which permits others to distribute, remix, adapt, build upon this work non-commercially, and license their derivative works on different terms, provided the original work is properly cited, appropriate credit is given, any changes made indicated, and the use is non-commercial. See: http://creativecommons.org/licenses/by-nc/4.0/.

\section{REFERENCES}

1. International Diabetes Federation. Diabetes: facts and figures. 2017. www.idf.org

2. Cheung N, Mitchell P, Wong TY. Diabetic retinopathy. The Lancet 2010;376:124-36.

3. Ding J, Wong TY. Current epidemiology of diabetic retinopathy and diabetic macular edema. Curr Diab Rep 2012;12:346-54.

4. Sivaprasad S, Gupta B, Crosby-Nwaobi R, et al. Prevalence of diabetic retinopathy in various ethnic groups: a worldwide perspective. Surv Ophthalmol 2012;57:347-70.

5. Cugati S, Wang JJ, Knudtson MD, et al. Retinal vein occlusion and vascular mortality: pooled data analysis of 2 population-based cohorts. Ophthalmology 2007;114:520-4.

6. Varma R, Bressler NM, Doan QV, et al. Prevalence of and risk factors for diabetic macular edema in the United States. JAMA Ophthalmol 2014;132:1334.

7. Emanuele N, Moritz T, Klein R, et al. Ethnicity, race, and clinically significant macular edema in the Veterans Affairs Diabetes Trial (VADT). Diabetes Res Clin Pract 2009;86:104-10.

8. Kaidonis G, Abhary S, Daniell M, et al. Genetic study of diabetic retinopathy: recruitment methodology and analysis of baseline characteristics. Clin Experiment Ophthalmol 2014;42:486-93.

9. Virgili G, Menchini F, Murro V, et al. Optical coherence tomography (OCT) for detection of macular oedema in patients with diabetic retinopathy. Cochrane Database Syst Rev 2011;7:CD008081.

10. Lima-Gómez V, Blanco-Hernández DM, Muñoz-lbarra P, et al. Severity distribution of diabetic macular edema at the time of diagnosis. Cir Cir 2011;79:491-7.

11. Davis MD, Bressler SB, Aiello LP, et al. Comparison of time-domain OCT and fundus photographic assessments of retinal thickening in eyes with diabetic macular edema. Invest Ophthalmol Vis Sci 2008;49:1745-52.

12. Brown JC, Solomon SD, Bressler SB, et al. Detection of diabetic foveal edema: contact lens biomicroscopy compared with optical coherence tomography. Arch Ophthalmol 2004;122:330-5.

13. Romero-Aroca P, Baget-Bernaldiz M, Fernandez-Ballart J, et al. Ten-year incidence of diabetic retinopathy and macular edema. Risk factors in a sample of people with type 1 diabetes. Diabetes Res Clin Pract 2011:94:126-32.

14. Kamoi K, Takeda K, Hashimoto K, et al. Identifying risk factors for clinically significant diabetic macula edema in patients with type 2 diabetes mellitus. Curr Diabetes Rev 2013;9:209-17.

15. Raman R, Rani PK, Kulothungan V, et al. Influence of serum lipids on clinically significant versus nonclinically significant macular edema: SN-DREAMS Report number 13. Ophthalmology 2010;117:766-72.

16. Knudsen LL, Lervang HH, Lundbye-Christensen S, et al. The North Jutland county diabetic retinopathy study (NCDRS) 2 . Nonophthalmic parameters and clinically significant macular oedema. $\mathrm{Br}$ J Ophthalmol 2007;91:1593-5.

17. Australian Census Data. 2011. http://www.censusdata.abs.gov.au/ census_services/getproduct/census/2011/quickstat/POA2170? opendocument\&navpos $=220$

18. Mitchell P. NHMRC Guidelines for the management of diabetic retinopathy: Department of Health and Ageing, 2008.

19. Stellingwerf C, Hardus PL, Hooymans JM. Two-field photography can identify patients with vision-threatening diabetic retinopathy: a screening approach in the primary care setting. Diabetes Care 2001;24:2086-90.
20. NHS Diabetic Eye Screening Programme. 2013. http://diabeticeye screening nhs uk/about

21. Anon. Grading diabetic retinopathy from stereoscopic color fundus photographs-an extension of the modified Airlie House classification. ETDRS report number 10 . Early treatment diabetic retinopathy study research group. Ophthalmology 1991;98(5 Suppl):786-806.

22. Bressler SB, Edwards AR, Chalam KV, et al. Reproducibility of spectral-domain optical coherence tomography retinal thickness measurements and conversion to equivalent time-domain metrics in diabetic macular edema. JAMA Ophthalmol 2014;132:1113-22.

23. Cruz-Herranz A, Balk LJ, Oberwahrenbrock T, et al. The APOSTEL recommendations for reporting quantitative optical coherence tomography studies. Neurology 2016;86:2303-9.

24. Wong TY, Klein R, Islam FM, et al. Diabetic retinopathy in a multi-ethnic cohort in the United States. Am J Ophthalmol 2006;141:446-55.

25. Kaidonis G, Abhary S, Daniell M, et al. Genetic study of diabetic retinopathy: recruitment methodology and analysis of baseline characteristics. Clin Exp Ophthalmol 2014;42:486-93.

26. Keenan TD, Johnston RL, Donachie PH, et al. United Kingdom National Ophthalmology database study: diabetic retinopathy; report 1: prevalence of centre-involving diabetic macular oedema and other grades of maculopathy and retinopathy in hospital eye services. Eye 2013;27:1397-404.

27. Yau JW, Rogers SL, Kawasaki R, et al. Global prevalence and major risk factors of diabetic retinopathy. Diabetes Care 2012;35:556-64.

28. Wang YT, Tadarati M, Wolfson $\mathrm{Y}$, et al. Comparison of prevalence of diabetic macular edema based on monocular fundus photography vs optical coherence tomography. JAMA Ophthalmol 2016;134:222-8.

29. Raymond NT, Varadhan L, Reynold DR, et al. Higher prevalence of retinopathy in diabetic patients of South Asian ethnicity compared with white Europeans in the community: a cross-sectional study. Diabetes Care 2009;32:410-5.

30. Benarous R, Sasongko MB, Qureshi S, et al. Differential association of serum lipids with diabetic retinopathy and diabetic macular edema. Invest Ophthalmol Vis Sci 2011;52:7464-9.

31. Klein R, Myers CE, Lee KE, et al. Oxidized low-density lipoprotein and the incidence of proliferative diabetic retinopathy and clinically significant macular edema determined from fundus photographs. JAMA Ophthalmol 2015;133:1054-61.

32. Hammes HP, Welp R, Kempe HP, et al. Risk factors for retinopathy and DME in Type 2 diabetes-results from the German/Austrian DPV Database. PLoS One 2015;10:e0132492.

33. Man RE, Sasongko MB, Wang JJ, et al. The association of estimated glomerular filtration rate with diabetic retinopathy and macular edema. Invest Ophthalmol Vis Sci 2015;56:4810-6.

34. Bergenstal RM, Tamborlane WV, Ahmann A, et al. Effectiveness of sensor-augmented insulin-pump therapy in type 1 diabetes. $N$ Engl $J$ Med 2010;363:311-20

35. Karges B, Schwandt A, Heidtmann B, et al. Association of insulin pump therapy vs insulin injection therapy with severe hypoglycemia, ketoacidosis, and glycemic control among children, adolescents, and young adults with type 1 diabetes. JAMA 2017;318:1358-66.

36. Lind M, Polonsky W, Hirsch IB, et al. Continuous glucose monitoring vs conventional therapy for glycemic control in adults with type 1 diabetes treated with multiple daily insulin injections: the GOLD randomized clinical trial. JAMA 2017;317:379-87.

37. Raman R, Gupta A, Kulothungan V, et al. Prevalence and risk factors of diabetic retinopathy in subjects with suboptimal glycemic, blood pressure and lipid control. Sankara Nethralaya diabetic retinopathy epidemiology and molecular genetic study (SN-DREAMS, Report 33). Curr Eye Res 2012;37:513-23.

38. Rooney D, Lye WK, Tan G, et al. Body mass index and retinopathy in Asian populations with diabetes mellitus. Acta Diabetol 2015;52:73-80.

39. Man REK, Sabanayagam C, Chiang PP-C, et al. Differential association of generalized and abdominal obesity with diabetic retinopathy in asian patients with Type 2 diabetes. JAMA Ophthalmol 2016;134:251-7. 